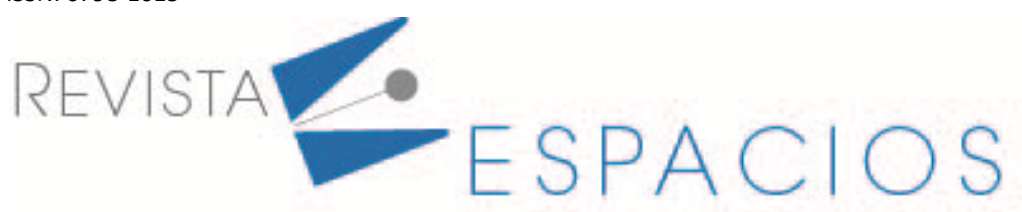

\title{
Análisis empírico de la competitividad para tres destinos tradicionales de sol y playa en Colombia
}

\section{Empirical analysis of the competitiveness for three traditional sun and beach destinations in Colombia}

\author{
MARIÑO, Juan P. ${ }^{1}$ \\ LEÓN, Martín ${ }^{2}$ \\ PINOCHET, Giselle ${ }^{3}$ \\ LANDINEZ, Natalia ${ }^{4}$
}

\section{Resumen}

Este estudio comparó los tres destinos turísticos de sol y playa más importantes de Colombia con el propósito de evaluar su competitividad turística. Aplicando el método multicriterio sobre el modelo de Crouch \& Ritchie, se consideraron 87 indicadores para valorar la dimensión política, económica, ambiental y social de Santa Marta, Cartagena y San Andrés. El método ELECTRE III arrojó datos sobre los cuales se incorporaron mejoras en la sistematización y análisis comparativos que ubicaron a Cartagena como el destino más competitivo del país.

Palabras clave: competitividad, turismo, análisis multicriterio, electre III

\begin{abstract}
This study compared the three most important sun and beach tourist destinations in Colombia in order to evaluate their tourist competitiveness. Applying the multicriteria method on the Crouch \& Ritchie model, 87 indicators were considered to assess the political, economic, environmental and social dimension of Santa Marta, Cartagena and San Andrés. The ELECTRE III method yielded data on which improvements were incorporated in the systematization and comparative analysis that placed Cartagena as the most competitive destination in the country.

Keywords: competitiveness, tourism, Multicriteria Analysis, electre III
\end{abstract}

\footnotetext{
${ }^{1}$ Docente investigador del programa de Administración de Empresas Turísticas y Hoteleras de la Fundación Universitaria UNITEC, miembro del grupo Gestión y Desarrollo Organizacional, reconocido y categorizado por Colciencias. Calle 76 \#12-58 Bogotá Colombia. Teléfono (+57 1) 7434344 . Correo <juanmarino@unitec.edu.co>

2 Profesor investigador del Departamento Económico-Administrativo, Unidad Regional Culiacán, Universidad Autónoma de Occidente, <http://orcid.org/0000-0002-8632-3833>. Blvd. Lola Beltrán km 1.5, Col. 4 de Marzo, 80020, Culiacán, Sinaloa. Tel: (667) 7591300, ext. 2356. Correo: martin.leon@uadeo.mx

${ }^{3}$ Docente investigadora de la Facultad de Ciencias Económicas y Administrativas de la Universidad Antonio Nariño, líder del grupo de investigación Innovación, Competitividad y Productividad de las Organizaciones, reconocido y categorizado por Colciencias. Calle 58 A BIS \#37 - 94 Teléfono: (+57 1) 315 2980. Correo: <gpinochet@uan.edu.co>

${ }^{4}$ Docente del programa de Administración Financiera del Instituto de Educación a Distancia (IDEAD), Universidad del Tolima, grupo de investigación GIETO reconocido por Colciencias. Calle 42 \# 1 - 01 Altos de Santa Helena, Ibagu\{e Colombia. Teléfono: (+57 ) 3177232039. Correo:

<nlandinezf@ut.edu.co>
} 


\section{Introducción}

Según la Organización Mundial del Turismo (UNWTO, por sus siglas en inglés), el turismo se ha convertido en una actividad económica de gran importancia para el desarrollo y crecimiento en las economías a escala mundial (OMT, 2018). Hasta antes de la llegada del Covid-19, la actividad turística aportaba el $10 \%$ del Producto Interno Bruto Mundial (PIBM) y era responsable del 7\% de las exportaciones mundiales, equivalentes a 1.6 billones de dólares estadounidenses al año, demás de generar 1 de cada 10 de los empleos que se ofrecían en orbe (OMT, 2017).

Datos del ente mundial dejan ver cómo 2018 cerró con un total de 1400 millones de turistas internacionales completando 6 años de crecimiento ininterrumpido (OMT, 2019), en los que 2017 se erigió como el año con mayor crecimiento en la llegada de turistas internacionales de la última década, con un aumento del $7 \%$ respecto al año anterior, debido a una demanda creciente motivada, en buena parte, por la normalización en la situación de orden público en algunos países en conflicto (OMT, 2018).

Para el caso de Latinoamérica, previo a la pandemia, la Comisión Económica para América Latina y el Caribe (CEPAL, 2018) daba cuenta de un crecimiento del orden del 5.6\% en las exportaciones por cuenta del turismo, a consecuencia, entre otras razones, de la devaluación de la monedas locales de la región respecto al dólar, lo cual incentivó la llegada de turistas internacionales y retuvo a los turistas domésticos. Para 2018, las Américas fueron la tercera región con mayor llegada de turistas internacionales, representando el $16 \%$ del total mundial, después de Europa con el $51 \%$ y Asia Pacífico con el 24\% (OMT, 2018). La llegada de turistas internacionales a la región aumentó en un 5\%, alcanzando los 211 millones de turistas internacionales y los 326000 millones de dólares estadounidenses al cierre de 2017.

Por su parte, Colombia ha desarrollado una considerable dependencia de los ingresos generados de la actividad turística, al punto de que, durante la mayor parte del siglo XXI, el turismo había sido reconocido como el segundo generador de divisas del país. De acuerdo con la Fundación Paz \& Reconciliación (PARES, 2018), Colombia venía presentando cifras positivas, sobresaliendo las logradas entre los años 2010 y 2015, quinquenio en el que la llegada de turistas internacionales aumentó un $70 \%$, la generación de divisas un $52 \%$, y el empleo un $17 \%$, producto del crecimiento en la cantidad de prestadores de servicios turísticos, además de un incremento en el Producto Interno Bruto (PIB) que alcanzó el 2.6\%.

\subsection{Competitividad de los destinos turísticos}

Para Jiménez \& De San Eugenio (2009), la actual situación de alta competitividad en los destinos turísticos ha creado la necesidad de evaluar todos aquellos aspectos que pudiera generar valor agregado para la atracción de potenciales viajeros, asegurando particularmente que la vocación de los destinos supere la mera aspiración y se fundamente sobre los pilares de las estructuras, infraestructuras y superestructuras. Y es que, según la OMT (2003), cuando se analiza la situación de los países latinoamericanos se aprecia que se siguen proyectando a futuro con sus esperanzas puestas en sus ventajas comparativas, con la expectativa de que la tierra siga proporcionando el sustento a sus habitantes, sin que dicho aprovechamiento incluya aspectos como la innovación, la gestión de sus gobiernos y la agregación de valor por los actores económicos locales para crear y poner a disposición de los mercados internacionales productos o servicios turísticos de clase mundial.

Por esta razón, ante la preeminencia de los recursos de índole natural y cultural, como factores de competitividad de los destinos turísticos, Diéguez et al. (2011), (refieriendo a Crouch \& Ritchie, 1999; Dwyer \& Kim, 2003; Hassan, 2000; Hu y Wall, 2005; Huybers y Bennett, 2003; Mihalič, 2000; Ritchie y Crouch, 2000, 2003, entre otros), destacan que la competitividad de los destinos turísticos sólo puede incrementarse mediante una gestión basada 
en la calidad medioambiental y en el desarrollo de un turismo sostenible que en palabras de Crouch (2007) redundan en la sostenibilidad.

Para Ritchie \& Crouch (2000), un destino turístico competitivo debe ser viable no únicamente en el plano económico, sino también en lo ecológico, social, cultural y político, puesto que más allá de propender por una derrama económica, producto de la explotación de los atractivos disponibles, deberá garantizar la sostenibilidad de los destinos si se quiere ser competitivo. No obstante, según Diéguez et al. (2011), (citando a Garau, 2006, y Crouch \& Ritchie 2000, 2003), fue el modelo de Calgary aquel que estableció un marco de referencia enfocado en mejorar el bienestar de la población con la explotación racional de los recursos en el marco de la sostenibilidad, erigiendo un escenario más específico cuando de referirse a la competitividad del turismo se trata.

Sobre el particular, es pertinente resaltar que, según Crouch (2007), la competitividad de los destinos turísticos ha motivado un número importante de investigaciones que se ha dividido en tres grupos. El primero reúne investigaciones cuyo objetivo fue medir la posición competitiva de destinos específicos alrededor del mundo, que incluyen Estados Unidos (Ahmed \& Krohn, 1990); Sun/Lost City, South África (Botha, Crompton \& Kim, 1999; Kim, Crompton \& Botha, 2000); turismo cultural en Toronto (Carmichael, 2002); Las Vegas (Chon \& Mayer, 1995); un casino resort (d'Hauteserre, 2000); Australia (Dwyer, Livaic \& Mellor, 2003); Hong Kong (Enright \& Newton, 2004); Asia Pacífico (Enright \& Newton, 2005); Canadian ski resorts (Hudson, Ritchie \& Timur, 2004); South Australia (Faulkner, Oppermann \& Fredline, 1999); Korea del sur y Australia (Kim, Choi, Moore, Dwyer, Faulkner, Mellor \& Livaic, 2001; Kim \& Dwyer, 2003); España y Turquía (Kozak, 2003; Kozak \& Rimmington, 1999); ciudades europeas (Mazanec, 1995); hoteles del Mediterráneo (Papatheodorou, 2002); suroeste de Asia (Pearce, 1997) y Zimbabwe (Vengesayi, 2005).

El segundo concentra aquello trabajos que midieron aspectos específicos de la competitividad de los destinos evaluando, entre otros, el posicionamiento del destino (Chacko, 1998), sistemas de gestión del destino (Baker, Hayzelden \& Sussmann, 1996), mercadeo del destino (Buhalis, 2000), competitividad de los precios (Dwyer, Forsyth \& Rao, 2000a, 2000b, 2000c, 2001, 2002; Stevens 1992; Tourism Council Australia 1998), calidad en la gestión (Go \& Govers 2000), el ambiente (Hassan, 2000; Mihalic, 2000), turismo basado en la naturaleza (Huybers \& Bennett, 2003), gerencia estratégica (Jamal \& Getz, 1996; Soteriou \& Roberts, 1998) y paquetes turísticos (Taylor, 1995).

Y el tercero, al que pertenece junto con Ritchie, en el cual se han desarrollado estudios enfocados en desarrollar modelos y teorías sobre la competitividad de los destinos turísticos. En este último grupo se inserta el presente manuscrito.

De acuerdo con el Reporte de Competitividad de Viajes y Turismo, publicado por el Foro Económico Mundial (WEF, por sus siglas en inglés), en 2015 Colombia empezó a presentar resultados positivos al ocupar el puesto 68 de entre 141 países evaluados (WEF, 2015), escalando 4 posiciones en un proceso que en 2007 la ubicaba en el puesto 72 de entre 124 países evaluados(WEF, 2007), y que finalmente le permitió llegar al puesto 55 entre 140 países evaluados (WEF, 2019), en el último reporte, antes de la llegada del coronavirus.

Datos del Ministerio de Comercio, Industria y Turismo (MINCUT, 2018), indicaron que en 2018 llegaron a Colombia más de 4.2 millones de visitantes no residentes, cifra que equivalía a un $8 \%$ de crecimiento respecto al año anterior, siendo Bogotá, Cartagena, Medellín y Cali las ciudades que recibieron más turistas internacionales hasta antes del confinamiento obligatorio. No obstante, San Andrés, Santa Marta y Cartagena son los destinos más destacados dentro de la tipología del turismo de sol y playa, por lo que el presente ejercicio de comparación permite determinar cuál de entre los tres destinos cuenta con mejores condiciones a nivel de estructuras, infraestructuras y superestructuras, que le otorguen mejores condiciones competitivas y, en consecuencia, mejores perspectivas de desarrollo local. 


\subsection{Destinos turísticos de sol y playa más relevantes en Colombia}

Al momento de hablar sobre el proceso evolutivo del turismo, la literatura especializada coincide en señalar que el desarrollo turístico estuvo circunscrito a ciudades costeras que a consecuencia del "boom del turismo" se consolidaron como enclaves tradicionales caracterizados por su evolución desde la pesca, el comercio y la agricultura, hacia la recepción de visitantes, quienes de manera masiva escogieron dichos destinos con la expectativa de disfrutar de sus playas (2012).

Según Benseny et al. (2012), tres cuartas partes de las ciudades más importantes del mundo se localizan a orillas del océano, por lo que las playas se han transformado en un recurso muy valorado, gracias a su importancia en el desarrollo turístico de las naciones. Para Alonso et al. (2003), Barragán (2003), Botero et al. (2008), Botero (2009), Cagilaba \& Rennie (2005), Cervantes (2008), James (2000), Yepes (1999) y Nelson et al. (2000), entre otros, es relevante la importancia de la actividad turística en las costas, pues su acelerado crecimiento en las últimas décadas ha impactado los sistemas sociales, ambientales y económicos de los destinos turísticos de sol y playa, comprometiendo su competitividad.

En esta medida, el turismo de sol y playa se convierte en uno de los productos que alcanza mayor generación de recursos económicos, pese a los impactos que causa en los ecosistemas con los que interactúa. Por ello resulta imperativo conocer los niveles de afectación que conlleva en las demás dimensiones de la sostenibilidad, en particular con la calidad de vida de los locales, el aspecto sociocultural y la misma capacidad del destino para responder con suficiencia a la demanda turística, en especial cuando esta se despliega dentro de enclaves masivos (Certificación de playa ambiental para hoteles; manejo integrado, 2019).

La experiencia internacional demuestra la dependencia que países como España, México, Brasil, Portugal, Cuba y California en Estados Unidos, tienen turísticamente de sus playas, no siendo una situación ajena a Colombia (Mariño, 2019), por lo que, en términos de competitividad turística, resulta imperativo analizar la competitividad de los tres principales destinos turísticos internacionales del país.

\subsubsection{Cartagena}

La ciudad de Cartagena se localiza al norte del departamento de Bolívar sobre la orilla del Mar Caribe. Se encuentra a 10 25' 30" latitud norte y 75 32' 25" de longitud oeste respecto al Meridiano de Greenwich (EduRed, 2020).

Cartagena, conocida como la "Ciudad Heroica», se distingue por su arquitectura colonial y por una intensa vida nocturna, festivales culturales y paisajes exuberantes. Ubicada en el mar Caribe, en sus playas se realiza el turismo; además, se caracteriza por su oferta gastronómica, acompañada de una importante infraestructura hotelera, lo que la hace uno de los destinos turísticos más visitados de Colombia (Colombia Travel, 2020a).

Reconocida por su patrimonio arquitectónico y cultural debido a su centro histórico, que se encuentra rodeado de antiguas murallas, declarada Patrimonio Histórico de la Humanidad por la Unesco en 1984 (Colombia, 2020). Destacan sus calles empedradas, logrando explorar el Castillo de San Felipe de Barajas, que data del siglo XVII. Además, sobresalen sus antiguas iglesias y sus monumentos famosos, como los dedicados a la India Catalina, a los Zapatos Viejos y a los Pegasos (Colombia Travel, 2020a).

Su población está compuesta por un millón 28 mil 736 habitantes (DANE, 2020), con una mayoría de composición étnica, resultado de la fusión de las tres etnias primigenias: la precolombina u originaria, la blanca, venida de España, y la negra, traída esclavizada de África (EduRed, 2020). 


\subsubsection{Santa Marta}

Ubicada frente al mar Caribe, se encuentra el destino de Santa Marta en el departamento de Magdalena al norte de Colombia. Identificado como el primer asentamiento español en Colombia, posee una extensión territorial de 2 mil 393 kilómetros cuadrados y con un área urbana de 55.1 kilómetros cuadrados, además de contar con clima promedio de 32 을 centígrados.

Reconocido oficialmente como Distrito Turístico, Cultural e Histórico, es vía de acceso para realizar excursiones al Parque Nacional Natural Tayrona, para los recorridos guiados a través del sitio arqueológico de la Ciudad Perdida en la Sierra Nevada de las montañas de Santa Marta, donde sobresalen las aguas cristalinas y arena blanca, lo que la hace ser considerada como una de las bahías más hermosa de América (Colombia Travel, 2020b).

Debido a la concentración de alrededor de 30 mil indígenas de las etnias Kogi, Arhuaco, Kankuamo y Wiwa, fue declarado en 1979 por la UNESCO como reserva de la Biósfera y patrimonio de la humanidad (Colombia Travel, 2020b). Destacan su infraestructura hotelera, que va de más 14 mil cuatros, distribuidos en 2 mil 673 establecimientos, compuestos desde hostales, departamentos, así como hoteles de lujo (Situr, 2020).

\subsubsection{San Andrés}

Con una extensión de 350 mil kilómetros cuadrados, localizado al noroccidente de Colombia, se encuentra el Archipiélago de San Andrés, Providencia y Santa Catalina, siendo este un grupo de Islas del Mar Caribe; es el único departamento de este país sin territorio continental. Entre sus características geográficas, este conjunto de islas, cayos, islotes y bancos, se localiza sobre una plataforma volcánica del Caribe suroccidental (San Andrés, 2020).

Debido a su ubicación en la zona intertropical, el archipiélago se caracteriza por las altas temperaturas que registran un promedio anual de $27.3^{\circ} \mathrm{C}$. Cuenta en su infraestructura turística con 5 mil 556 habitaciones, distribuidas en 451 establecimientos de hospedaje (San Andrés, 2020), el cual incluye hoteles resort, boutique, así como posadas nativas, que sirven como alojamiento turístico. Fue declarado en el año 2000 Reserva Mundial de la Biósfera Seaflower.

Las influencias de ingleses, españoles, piratas y corsarios se mezclaron con el pueblo afro para dar como resultado una cultura de mucha riqueza. Es común escuchar a los lugareños hablar creole, una particular mezcla de inglés isabelino, español y dialectos africanos. Por ello, el Archipiélago fue escogido como el mejor destino de Sol y Playa en los World Travel Awards de 2014, 2015 y 2019 (Colombia Travel, 2020c).

\section{Marco téorico}

Existen estudios desarrollados de la competitividad del destino. Sin embargo, son escasos los que están desarrollados con métodos MCDA. En esta sección se explican los modelos de competitividad del turismo y obras relevantes desarrolladas con métodos MCDA.

\subsection{Análisis de los modelos de competitividad de turismo}

El concepto competitividad puede estudiarse desde diferentes ángulos: mediante productos, de las empresas, de las ramas de la economía o de las economías nacionales y locales, así como en el corto, mediano o largo plazo. A pesar de la extensa literatura sobre competitividad, hoy día no se dispone de un consenso o definición clara para la discusión; por ende, tampoco acerca de cómo medirla (Benzaquen et al., 2010; Gomezelj, 2006).

Las definiciones que ofrece la literatura proporcionan una micro y macroconnotación de lo que es la competitividad (Gomezelj, 2006; Crouch, 2010). Desde una perspectiva macro, porque la competitividad es de preocupación nacional y su objetivo es mejorar los ingresos reales de la comunidad, y desde la perspectiva micro, 
porque esta es estudiada a la escala de una empresa, ya que es aquí donde toda organización debe proporcionar productos y servicios, los cuales deberán satisfacer los deseos del consumidor moderno.

En este sentido, Porter (1991) afirma que la competitividad de una nación depende de la capacidad de sus industrias para innovar y mejorar, por lo que determinadas empresas son capaces de hacerlo con coherencia, procurando las mejoras y cada vez más perfeccionada de la ventaja competitiva. Y Krugman (1994) sostiene que la competitividad pierde relevancia en el ámbito nacional, ya que los principales países no están compitiendo entre ellos, por lo que se trata más bien de un asunto interno de la nación que de un aspecto externo.

Duque (2005) define la competitividad como la capacidad de competir, ya que en el contexto internacional la competitividad se refiere a la aptitud de competir en los mercados, siendo esta la capacidad de un sector de colocar con éxito sus productos en el mercado internacional, en condiciones de libre competencia. Y Labarca (2008) destaca que la competitividad de una industria puede medirse en términos de la rentabilidad general de las empresas, la balanza comercial en la industria, el balance entre inversiones extranjeras directas salientes y entrantes y mediciones directas de costo y calidad, considerando que la competitividad en la industria es un indicador de la fortaleza económica de un país.

A este interés por estudiar la competitividad se agrega el estudio de la competitividad de los destinos turísticos, resultado de los aportes específicos de autores como Porter (1998), Crouch y Ritchie (1999), Dwyer et al. (2004), Ritchie y Crouch (2003), Dwyer y Kim (2003), Duque (2005), Ascanio (2008), Díez (2010) y Crouch (2010), entre otros. En el sentido de que la competitividad se ha convertido en un asunto de importancia de orden económico para aquellos países donde el turismo se ha constituido como un importante generador de divisas y que aporta al desarrollo económico.

Crouch y Ritchie (1999), como un esfuerzo en sus investigaciones, han tratado de desarrollar modelos y teorías generales de competitividad de los destinos que no son específicos para determinados destinos o atributos. Estos autores empezaron a estudiar la naturaleza y estructura de la competitividad del destino (Crouch y Ritchie, 1999; Ritchie y Crouch, 2003). Su objetivo era desarrollar un modelo conceptual basado en las teorías de la ventaja comparativa de Smith y Ricardo y la ventaja competitiva (Porter, 1998), adaptado a las características distintivas de la competencia del destino.

En el desarrollo del modelo, Crouch (2010) incluye conceptos genéricos que derivan en un modelo que postula la competitividad del destino turístico, determinado por cinco componentes principales: calificadores y amplificadores determinantes, política del destino, planeación y desarrollo, gestión del destino, factores y recursos de atracción y los factores condicionantes y limitadores. Es común observar que la iteración del visitante se basa en los factores y recursos de atracción, ya que incluye los elementos primarios de recurso del destino. Y son estas algunas de las razones fundamentales por las cuales los posibles visitantes eligen un destino sobre otro (Crouch y Ritchie, 1999, p. 146).

De esta manera, estos factores y recursos de atracción constituyen los elementos principales del recurso de destino e incluyen la fisiografía, la cultura y la historia, las relaciones de mercado, actividades especiales y la superestructura turística. Fisiografía abraza el paisaje y el clima; los vínculos de mercado incluyen los vínculos con los residentes de las regiones turísticas de origen, y la superestructura turística se compone principalmente de las instalaciones de alojamiento, servicios de comida, servicios de transporte y las principales atracciones.

Competitivamente, el turismo en los estudios de Crouch (2010) determina que la gestión de la competitividad del destino se ha convertido en un tema de interés, debido a las teorías, modelos y procesos que pueden ayudar a guiar el enfoque en este reto, ya que ofrecen la posibilidad de posicionar con claridad y rigor una tarea compleja de gestión. Y extienden su análisis Crouch en colaboración con Ritchie (Ritchie y Crouch, 2003), quienes la definen 
como la capacidad relativa de un destino para satisfacer las necesidades de los visitantes en los diversos aspectos de la experiencia turística.

En relación con este modelo propuesto por Crouch y Ritchie, se gestaron investigaciones como las desarrolladas por Crouch (1995), Kim y Dwyer (2003), Sánchez et al. (2004), Dwyer et al. (2004), Duque (2005), Gomezelj (2006) y Gándara et al. (2013), entre otros. Por lo que la literatura en relación con el modelo propuesto por los autores en análisis se verá favorecida a través de más investigaciones en profundidad sobre los factores específicos o categorías que permiten medir la competitividad de los destinos.

En cuanto a los modelos que analizan los factores que pueden determinar si un destino es más competitivo en relación con otro, hay algunos enfoques centrados en la competitividad del destino. En esta sección se describen los enfoques propuestos por Porter (1998) y Crouch (2010), además de destacar la definición de competitividad de Dwyer y Kim (2003).

El enfoque de Porter (1998) no se centra en el estudio de las empresas. Más bien, se estudian países o destinos; en su principal idea, distingue que el éxito de una empresa depende no sólo de la estrategia y el posicionamiento, sino también sobre el medio ambiente. También considera destinos como «clústers», debido al éxito de un sector en particular, donde el ambiente es dinámico, desafiante y anima a las empresas a mejorar su ventaja.

Por otro lado, Crouch (2010) afirma que «el producto turístico es una experiencia que se entrega por un destino para los visitantes». Esto significa que la complejidad adicional del producto en sí consiste en un mayor número de atributos, lo que garantiza que cada visitante se lleva a casa una experiencia única. Al mismo tiempo, existen objetivos diferentes que están detrás de la política del turismo privado y público. Mientras que algunos de los objetivos abordan el rendimiento económico y el beneficio, otros objetivos se refieren al contexto ambiental y social.

Crouch (2010) señala que la gestión de la competitividad del destino se ha convertido en un tema de interés debido a las teorías, modelos y procesos que pueden orientar el enfoque a este desafío, ya que ofrecen la posibilidad de lograr con claridad y rigor una tarea de compleja gestión. De esta manera, impresiona que el impacto de un atributo de la competitividad en el rendimiento relativo de un destino es una función, siempre y cuando la importancia del atributo sea variable en el destino.

Este modelo Crouch-Ritchie (2010) se acentúa principalmente por dos factores que reconocen la complejidad del destino, que se basa en la dotación de recursos de un destino (ventaja comparativa) y en su capacidad de desplegar recursos (ventaja competitiva) y obtener el impacto del entorno macroeconómico y microeconómico.

Por último, la competitividad de los destinos según Dwyer y Kim (2003) se puede definir como «la capacidad relativa de un destino para satisfacer las necesidades de los visitantes en los diversos aspectos de la experiencia turística».

\subsection{Análisis empíricos de la competitividad con el método multicriterio}

Respecto a estudios de Competitividad con métodos de Análisis Multicriterio para la Toma de Decisiones (MCDA, por sus siglas en inglés), Peng y Tzeng (2012) exploraron estrategias para mejorar la competitividad del turismo implementando un modelo MCDM y combinando DEMATEL -basado ANP. Álvarez, León, Gastélum y Vega (2013), hicieron un análisis empírico de la competitividad de las ciudades de Sinaloa, México, con el método de outranking. Leyva, Gastélum y Urías (2013), desarrollaron la aplicación de un enfoque multicriterio para comparar sectores económicos: el caso del estado de Sinaloa, México.

A su vez, León y Leyva (2017) exponen un análisis de la competitividad de destinos tradicionales de sol y playa en México, basado en el método multicriterio. León y Leyva (2016) plantean una ayuda de decisión multicriterio 
para evaluar la competitividad de los destinos turísticos en el noroeste de México. Pérez et al. (2018) presentan un análisis comparativo de la competitividad de los destinos turísticos Querétaro y Guanajuato bajo un enfoque multicriterio, y León, Flores y Leyva (2019), Determinación de la Competitividad de los Destinos Turísticos Bajo un Enfoque Multicriterio en el Noroeste De México. Un Análisis Comparativo 2015-2017.

Con estos estudios empíricos, se encontró que es posible con el modelo de Crouch y Ritchie explicar los niveles de actividad turística mejor que el crecimiento del turismo sostenido. Además, realizaron recomendaciones sobre la forma de ajustar la estrategia de futuro de la investigación sobre la competitividad de los destinos turísticos.

Finalmente, tomando en consideración la amplitud y variedad de los indicadores que utilizan para medir la competitividad turística utilizados en la literatura, es evidente que las decisiones a las que se enfrentan los planificadores de turismo suelen incluir variables que son difíciles de medir directamente, e incluso, si todas las variables se pueden medir con precisión aún existen graves problemas que se plantean para obtener medidas numéricas de la importancia relativa de las variables de decisión. (Crouch, 2010).

\section{Metodología}

La presente investigación, en la que se busca determinar la competitividad de los destinos turísticos de una región geográfica, pasa al uso de los números para medir y expresar las propiedades del universo social y natural en forma cuantitativa precisa, adicional a las decisiones a las que se enfrentan los planificadores de turismo y que suelen incluir variables difíciles de medir directamente; es en este sentido en que para el alcance de los objetivos, por tratarse de un estudio en que son analizadas las variables involucradas en el problema, ésta es de tipo cuantitativo mediante un estudio de caso (León, 2016).

Adicionalmente, por ser un modelo estrechamente relacionado con la creación de entornos simulados para el estudio de situaciones hipotéticas, o para crear sistemas de gestión que permitan disminuir la incertidumbre. permite que las mismas entradas produzcan las mismas salidas, eliminando el azar y la incertidumbre. De esta manera, la inclusión de una mayor complejidad en las relaciones con un número mayor de variables hará posible que éste se aproxime a un modelo probabilístico (León, 2016).

Asimismo, y en virtud de que no hay antecedente científico que demuestre un estudio de estas características en Colombia, ya que en éste se involucra la especificación de un conjunto $A$ de soluciones de alternativas factibles. En este sentido, se realiza la manipulación de variables dentro de la experimentación de los modelos utilizados, como es la ayuda a la decisión multicriterio (Multicriteria Decision Aids [MCDA]), la cual asume que los atributos y criterios asignan una descripción a las alternativas, que puede ser cuantitativa o cualitativa (León y Leyva, 2016).

Por tanto, esta investigación se basa en el modelo de competitividad desarrollado por Crouch y Richie (2010), que presentan una lista de 36 atributos, los cuales pueden ser datos cualitativos o cuantitativos, y 5 criterios para evaluar la competitividad de los destinos. En este sentido, adaptamos este modelo a este problema cambiando algunos atributos y eliminando otros, como son eventos especiales, fisiografía y clima, cultura e historia, mezcla de actividades, entretenimiento, superestructura, ubicación, infraestructura, costo/valor, interdependencias, sensibilización/imagen y capacidad de carga, y se añadieron atributos más regionales y que no fueron considerados en su trabajo, éstos son: ferias y festivales, restaurantes, cuartos y unidades de hospedaje, cafeterías, centros nocturnos, parques y balnearios, campos de golf.

El modelo final de competitividad que se plantea para este estudio incorpora 87 atributos, que se agruparon en 4 dimensiones o factores: Gestión del Destino, Factores y Recursos de Atracción, Factores Ambientales y Factores Sociales. En este análisis, se busca realizar un comparativo de los tres destinos de sol y playa más importantes 
de Colombia, con el propósito de evaluar su realidad competitiva, y a partir de la misma identificar su potencial de desarrollo local. Para ello, las principales técnicas de recolección de datos, utilizadas en esta investigación, correspondieron a la búsqueda de información a través de fuentes documentales (o secundarias), entrevista a expertos y la generación de indicadores compuestos mediante simulación por computadora.

En este sentido, entre otras fuentes de información, se destacan los resultados del Censo Nacional de Población y Vivienda 2018 (DANE), de las oficinas de turismo de las gobernaciones de Santa Marta, Cartagena y San Andrés, CITUR, SITUR, Ministerio de Educación Nacional de Colombia y del Viceministerio de Turismo, considerándose fuentes fiables, generadoras de información en relación con el turismo y sus actividades derivadas, con pertinencia para los propósitos del tema investigado.

Finalmente, al abordar el método cuantitativo, se hicieron una serie de ejercicios, a manera de prueba piloto, y se consideró trabajar con decisores reales, lo que permitió utilizar el enfoque constructivo, propio de la ayuda a la Decisión Multicriterio (MCDA) (Leyva, 2010), el cual contempla la figura de un analista que ayuda al decisor a construir sus propios modelos de racionalidad con base en sus respuestas a las preguntas relacionadas con las preferencias, para lo cual se diseñó el instrumento para esta finalidad.

\subsection{Metodología de análisis multicriterio}

Por tanto, este análisis se basó en el método multicriterio sustentado en el modelo planteado por Crouch-Ritchie (1999), el cual fue desarrollado para el análisis de la competitividad de los destinos turísticos; en él se consideran los atributos establecidos para realizar la misma comparación, adicional a que se agrupan en cuatro criterios, siendo estos utilizados en el método de ordenamiento, mismo que incorpora mejoras sobre los métodos tradicionales de comparación (León y Leyva, 2017).

En este sentido, el enfoque multicriterio se presenta como una herramienta fundamental para la investigación, debido a que ayuda a implementar técnicas de comparación multidimensional basados en la informática. Para el caso particular, se emplea el procedimiento propuesto por León y Leyva (2019) para determinar la competitividad de los destinos turísticos, que sugieren el uso del método ELECTRE III propuesto por Roy (1991), como instrumento para el procesamiento y cálculo.

Comparado con otros métodos, como el de componentes principales (CP), se opta por el ELECTRE III, dado que el primero acusa falta de claridad a la hora de incluir el juicio de los expertos durante el proceso de toma de decisiones, además de que algunos criterios pueden tener una estructura cualitativa incierta que no se puede medir con precisión, lo que no sucede en el uso de ELECTRE III, ya que desde el planteamiento del problema se vislumbra el uso de variables cualitativas y se definen los valores que éstos tomarán.

Por ello, en esta sección se resuelve un problema de clasificación multicriterio con el objetivo de determinar la competitividad turística. Por lo que se consideran tres destinos para clasificarlos en orden descendente. El método utilizado es el ELECTRE III y se basa en el enfoque de Bartolini, Gallerani, Samoggia y Viaggi (2005), quienes hacen una propuesta para llevar a cabo el proceso de análisis, que puede utilizarse con fines de toma de decisiones. Por tanto, el enfoque de análisis multicriterio requiere dos fases: 1) la construcción del modelo y 2) el uso del modelo. 
Figura 1

Esquema de ayuda a la Decisión Multicriterio (MCDA)

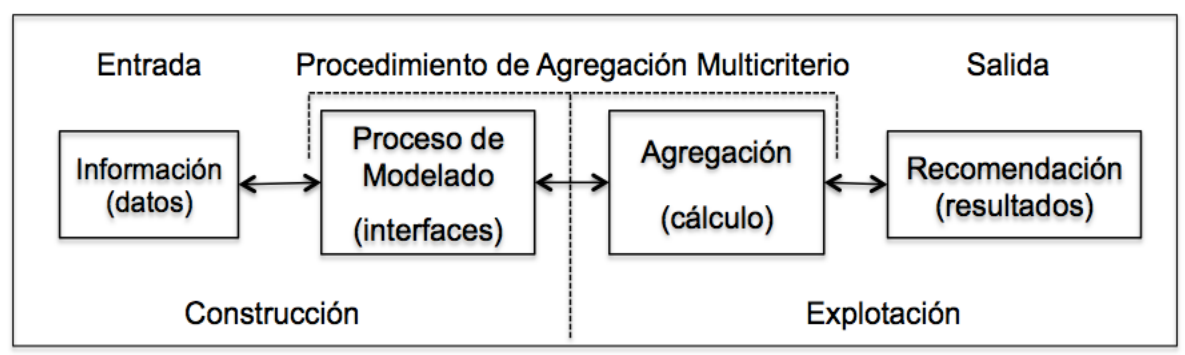

Fuente: Elaborado con base en Bartolini et al., 2005.

Este procedimiento acorta el proceso de apoyo a la toma de decisiones multicriterio, que es el de indicar con mayor detalle este enfoque, mismo que se ejecuta a través de una serie de pasos identificados de la siguiente manera:
a) Definición del problema
b) Identificación de alternativas
c) Escala de medición y caracterización de los criterios
d) Proceso de normalización de las variables
e) Determinación de pesos
f) Parámetros de indiferencia y preferencia
g) Construcción de la matriz de rendimiento, y
h) Orden final

En la definición del problema, Bartolini et al. (2005) señalan que el primer paso para el análisis multicriterio es establecer el problema en términos de los objetivos de la evaluación, asume como sección importante la interacción entre el analista y el responsable de la toma de decisiones, y considera que para una evaluación correcta es importante elegir la metodología coherente con los siguientes aspectos:

- El papel de la decisión en la evaluación

- Su nivel de complejidad y transparencia

- Información disponible

En la identificación de alternativas: se construye una matriz de alternativas. Donde $\boldsymbol{A}=\left\{\boldsymbol{a}_{1}, \boldsymbol{a}_{2}, \ldots, \boldsymbol{a}_{j}, \ldots, \boldsymbol{a}_{\boldsymbol{m}}\right\}$, es el conjunto finito de alternativas, $|\boldsymbol{A}|=\boldsymbol{m}$.(2006).

Escala de medición y caracterización de los criterios: para cada criterio se propone una serie de indicadores $\left(\boldsymbol{C}_{\boldsymbol{j}}\right)\left(\boldsymbol{x}_{1}, \boldsymbol{x}_{2}, \ldots, \boldsymbol{x}_{\boldsymbol{n}}\right)\left(\boldsymbol{a}_{\boldsymbol{i}}\right)\left(\boldsymbol{C}_{1}, \boldsymbol{C}_{2}, \ldots, \boldsymbol{C}_{\boldsymbol{n}}\right)$ en un grupo de destinos, a partir de éstos, se calcula un conjunto de criterios. Cada uno donde hay una combinación de originales por peso para cada criterio (Almeida, Figueira y Roy, 2006), es decir: $\left(C_{j}\right)(j=1, \ldots, n) x_{1}, x_{2}, \ldots, x_{n}\left(w_{j}\right)$.

$$
C_{j}\left(a_{i}\right)=\left(w_{j} * x_{j}\left(a_{i}\right)\right)+\left(w_{2} * x_{2}\left(a_{2}\right)\right)+\cdots+\left(w_{n} * x_{n}\left(a_{n}\right)\right)
$$

Proceso de normalización de las variables: este procedimiento tiende a encontrar que la escala normalizada cubre el rango entre 0 y 1 de los valores a reescalado (Barba-Romero, 1987), de la siguiente manera: 


$$
\operatorname{Vn}\left(a_{i}\right)=\frac{x\left(a_{i}\right)-\operatorname{Min}}{\operatorname{Max}-\operatorname{Min}}
$$

Dónde:

$$
\begin{aligned}
& \operatorname{Vn}\left(a_{i}\right)=\text { valor normalizado } \\
& x\left(a_{i}\right)=\text { valor del indicador } \mathrm{i} \\
& \text { Valor mínimo del indicador } \mathrm{i}, \mathrm{y} \\
& \text { Max- Valor máximo del indicador } \mathrm{i}
\end{aligned}
$$

La asignación de pesos: para indicadores individuales a través de la matriz de comparación, consiste en construir un (nxn) de variables. (Alireza, Majid y Rosnah, 2010) y los pesos para los criterios se determinan a través de la teoría de la construcción personal: Donde wj es el coeficiente de importancia relativa otorgado al criterio gj, propuesto por Roger, Bruen y Maystre (2000).

Parámetros de indiferencia y preferencia: uno de los apoyos proporcionados al responsable de la toma de decisiones es la definición de preferencias e incertidumbres a través de los umbrales de indiferencia (q), preferencia $(p)$ y veto $(v)$.

- $\quad q_{j}\left(g_{j}((a))=a_{j} * g_{j}(a)+b_{j}\right.$ : el umbral de indiferencia de acción directa de una comparación con la acción $b$, cuando las preferencias están en la dirección de aumento y, $g_{j}(a)<g_{j}(b)$ paraj $=1,2 \ldots, n$;

- $\quad p_{j}\left(g_{j}((a))=a_{j} * g_{j}(a)+b_{j}\right.$ umbral de preferencia directa de una acción en comparación con la acción b, cuando las preferencias están en la dirección creciente y $g_{j}(a)<g_{j}(b)$, paraj $=1,2 \ldots, n$;

- $\quad v_{j}\left(g_{j}((a))=a_{j} * g_{j}(a)+b_{j}\right.$ el umbral de veto directo de la acción de una comparación con la acción $\mathrm{b}$, cuando las preferencias están en la dirección del aumento $\mathrm{y} g_{j}(a)<g_{j}(b)$, para $j=1,2 \ldots, n$; (Almeida et al. 2006).

Construcción de la matriz de rendimiento: las ciudades que son evaluadas con los criterios permiten generar una matriz de rendimiento, que se construye con alternativa $m$ de mágicas por los cuatro criterios de decisión.

Con esta secuencia, se puede establecer un orden en el desarrollo del problema, desde su argumentación hasta su conclusión, donde una de las características básicas del análisis multicriterio es comparar alternativas basadas en una serie de criterios. Por tanto, la prueba para aceptar la aserción de aSb se implementa utilizando dos principios:

El principio de concordancia exige que la mayoría de los criterios, después de considerar su importancia relativa, estén a favor de la afirmación -el principio de la mayoría- (Leyva, 2010). Es decir, el primer paso consiste en desarrollar una medida de acuerdo que se incluya en el índice de concordancia, para cada par de alternativas, por lo que una relación de $C(a, b)$ nivel superior difusa se define de la siguiente manera:

$$
c(a, b)=\frac{1}{W} \sum_{j=1}^{n} c_{j}(a, b) \text { where } W=\sum_{j=1}^{n} w_{j}
$$




$$
C_{j}(a, b)=\left\{\begin{array}{c}
1 \text { if } g_{j}(a)+q_{j}\left(g_{j}(a)\right) \geq g_{j}(b) \\
0 \text { if } g_{j}(a)+p_{j}\left(g_{j}(a)\right) \geq g_{j}(b) \\
\text { linearly increasing with } g_{j}(a) \text { in the intermediate region }
\end{array}\right.
$$

El principio de discordia se llama umbral de veto. El umbral de veto permite rechazar la posibilidad de rechazarla en su totalidad -el respeto de los principios de las minorías- (Leyva, 2010) se calcula como: $v_{j} a S b$

$$
d_{j}(a, b)=\left\{\begin{array}{c}
1 \text { if } g_{j}(a)+p_{j}\left(g_{j}(a)\right) \leq g_{j}(b) \\
0 \text { if } g_{j}(a)+v_{j}\left(g_{j}(a)\right) \leq g_{j}(b) \\
\text { linearly increasing with } g_{j}(a) \text { in the intermediate region }
\end{array}\right.
$$

Por último, El principio de discordia exige que dentro de la minoría de criterios que no sean compatibles con la afirmación ninguno de ellos se oponga firmemente a la afirmación: el respeto de los principios de las minorías (Leyva, 2010).

\section{Resultados: análisis de la competitividad del destino mediante el Método Outranking}

El problema descrito fue tratado con el sistema de apoyo a la decisión multicriterio (SADGAGE) (Leyva y Fernández, 2003), este sistema SADGAGE fue diseñado basado en la metodología multicriterio para el problema de clasificación que utiliza ELECTRE III (ELimination Et Choix Traduisant la REalité) (Roy, 1990), el cual modela las preferencias con un y un algoritmo evolutivo multiobjetivo (MOEA) (Leyva y Aguilera, 2005).

Figura 2

Configuración de un proyecto de clasificación multicriterio en Sadgage

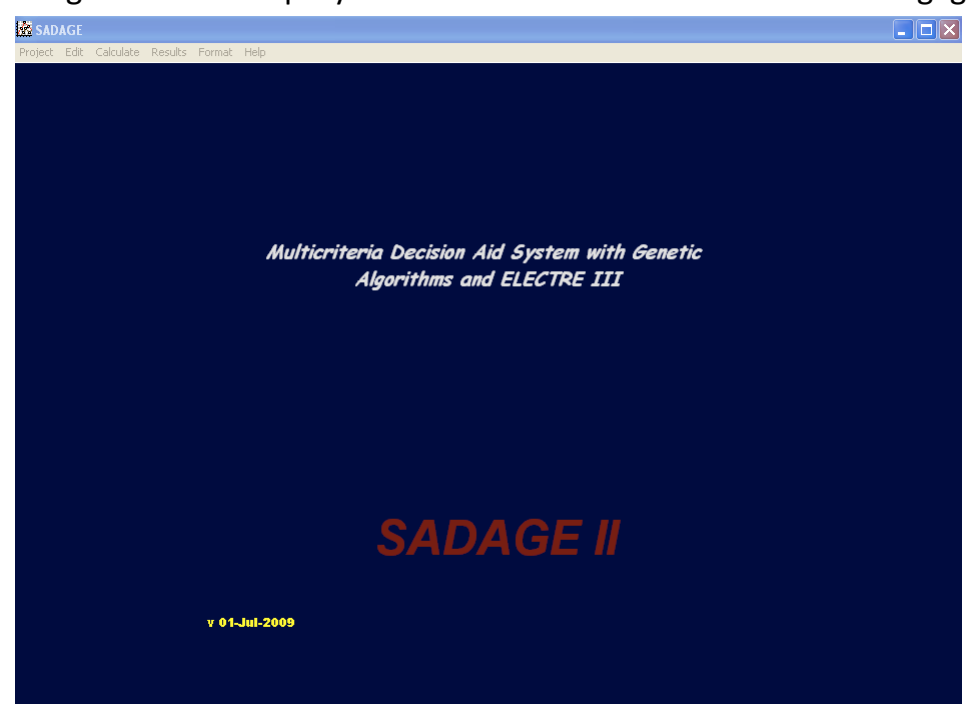

Fuente: Software Multicriteria decisión Aid System - SADAGE II (2020).

Por su parte, el estudio está basado en el modelo de Crouch y Ritchie (2010) y utilizado para medir la competitividad de los destinos turísticos del noroeste de México (León y Leyva, 2017); se clasificó para tres destinos turísticos de Colombia, con el objetivo de encontrar el destino más competitivo. éstos corresponden a los de sol y playa ubicados en las costas del Mar Caribe, como son San Marta, Cartagena y San Andrés (cuadro $1)$. 
Cuadro 1

Destinos por evaluar

\begin{tabular}{|c|c|}
\hline Código & Destino \\
\hline$A_{1}$ & Santa Marta \\
\hline$A_{2}$ & Cartagena \\
\hline$A_{3}$ & San Andrés \\
\hline
\end{tabular}

Fuente: Elaboración propia, 2020.

Por su parte, el modelo conceptual de competitividad del destino planteado por Crouch y Ritchie (2010) incorpora una lista de atributos que permiten evaluar la competitividad de los destinos, por lo que se adaptaron al modelo y se modificaron algunos atributos. En este sentido, en el modelo (tabla 2) se consideran los criterios para evaluar y en el cuadro 3 se presentan los valores de cada alternativa (destino) respecto a cada criterio, denominada como matriz de desempeño.

\section{Cuadro 2}

Criterios de los destinos a evaluar

\begin{tabular}{|c|c|}
\hline Código & Criterios \\
\hline $\mathrm{C}_{1}$ & Gestión del Destino \\
\hline $\mathrm{C}_{2}$ & Factores y Recursos de Atracción \\
\hline $\mathrm{C}_{3}$ & Factores Ambientales \\
\hline $\mathrm{C}_{4}$ & Factores Sociales \\
\hline
\end{tabular}

Fuente: Elaboración propia, 2020.

Cuadro 3

Matriz performance de las alternativas

\begin{tabular}{|c|c|c|c|c|}
\hline & $\mathrm{C}_{1}$ & $\mathrm{C}_{2}$ & $\mathrm{C}_{3}$ & $\mathrm{C}_{4}$ \\
\hline $\mathrm{A}_{1}$ & 12.08 & 16.84 & 5.63 & 13.84 \\
\hline $\mathrm{A}_{2}$ & 17.77 & 13.17 & 6.80 & 14.49 \\
\hline $\mathrm{A}_{3}$ & 3.42 & 8.77 & 1.45 & 3.20 \\
\hline $\mathrm{A}_{4}$ & 12.08 & 16.84 & 5.63 & 13.84 \\
\hline
\end{tabular}

Fuente: Elaboración propia, 2020.

Con base en la metodología ELECTRE III (Roy, 1991), a cada criterio se le aplicaron opciones de pesos, umbrales de indiferencia y umbrales de preferencia, sin considerar el umbral de veto (cuadro 4).

\section{Cuadro 4}

Parámetros de los criterios; pesos, umbrales de indiferencia y de preferencia

\begin{tabular}{|c|c|c|c|c|}
\hline & $C_{1}$ & $C_{2}$ & $C_{3}$ & $C_{4}$ \\
\hline$w$ & 0.33 & 0.33 & 0.25 & 0.09 \\
\hline$q$ & 4 & 7 & 1 & 2 \\
\hline$p$ & 15 & 15 & 5 & 11 \\
\hline
\end{tabular}

$w$ es el peso de los criterios.

$q$ es el umbral de la indiferencia de los criterios.

$p$ es el umbral de la preferencia de los criterios

Fuente: Elaboración propia, 2020.

De acuerdo con lo señalado, para la toma de decisiones se utilizó el método ELECTRE III para construir una relación outranking. Posteriormente, se utilizó el algoritmo evolutivo presentado en Leyva y Aguilera (2005) para explotar la relación de superación y derivar una clasificación final de las alternativas. 
Con la información proporcionada, se construyó un modelo de integración de preferencias de las alternativas sintetizado como una relación de superación borrosa; para este propósito, se utilizó el principio de concordancia y discordancia, como se concibe en ELECTRE III (cuadro 5).

\section{Cuadro 5}

Matriz de credibilidad

\begin{tabular}{|c|c|c|c|}
\hline \multicolumn{5}{|c|}{ Matriz de credibilidad } \\
\hline & A1 & A2 & A3 \\
\hline$A_{1}$ & 1.00 & 1.00 & 1.00 \\
\hline$A_{2}$ & 1.00 & 1.00 & 1.00 \\
\hline$A_{3}$ & 0.97 & 0.93 & 1.00 \\
\hline
\end{tabular}

Fuente: Elaboración propia, 2020.

Obtenido el modelo de preferencias del decisor (matriz de credibilidad), el siguiente paso es su explotación con el fin de obtener un ranking de alternativas. Para ello, se utilizó un método basado en el algoritmo evolutivo multiobjetivo (MOEA), presentado en Leyva y Aguilera (2005).

Como se ha mencionado, se utilizó el software SADGAGE, y se utilizó el MOEA para obtener un ranking de alternativas, con los parámetros definidos que se muestran de la siguiente manera:

- Número de generaciones: 10000

- Tamaño de la población: 30

- Probabilidad: 0.95

- La mutación de probabilidad: 0.05 .

A medida que cada posición en el orden ha asignado un $w_{i}$ peso, se calcula la suma ponderada como a continuación se presenta:

$$
\sum_{i=1}^{m} w_{i} T(i, j), j=1 . ., m
$$

con el que se obtuvo la recomendación final a la que toma las decisiones. Como se ha señalado, las alternativas en todo momento mantuvieron su posición en el ranking con un nivel de credibilidad de 0.9899, que está cerca de 1.0 da mayor certeza de los resultados. 
Figura 3

Muestra qué posiciones obtuvieron cada alternativa del proyecto durante las ejecuciones del Algoritmo Genético

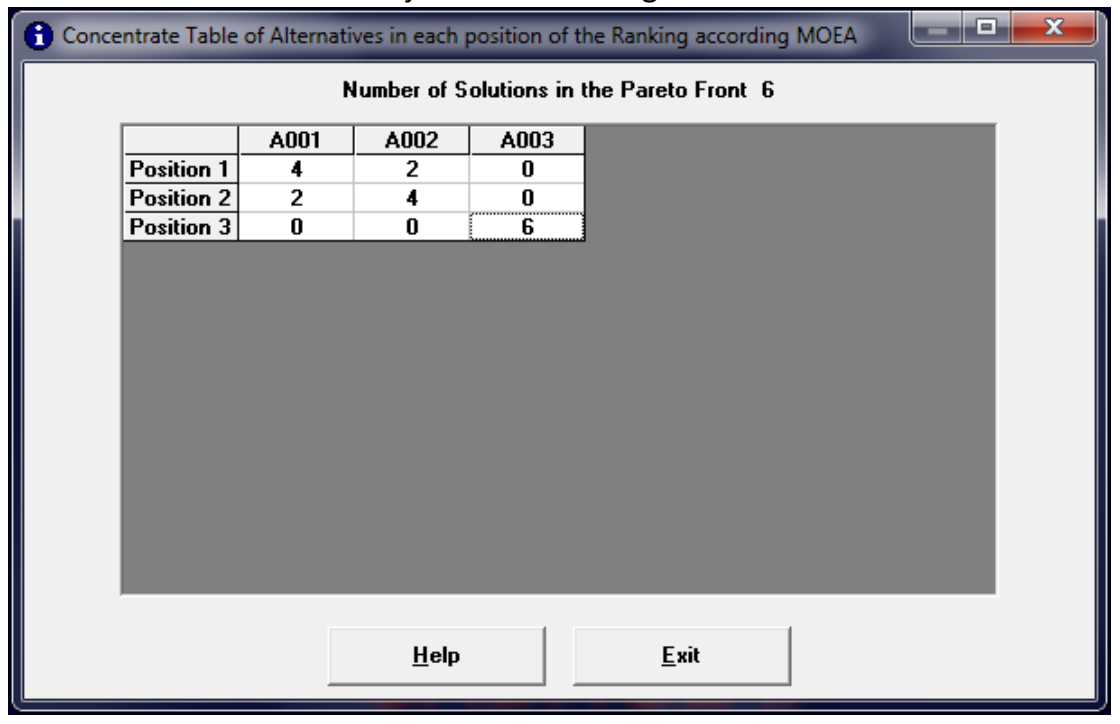

Fuente: Software Multicriteria decisión Aid System - SADAGE II (2020).

Finalmente, el cuadro 6 sugiere la clasificación final del ordenamiento, en la que se destaca Cartagena como el destino más competitivo, seguido por Santa Marta y San Andrés.

\section{Cuadro 6}

Ranking de alternativas

\begin{tabular}{|c|c|}
\hline Posición & Destino \\
\hline 1 & $\mathrm{~A}_{2}$ \\
\hline 2 & $\mathrm{~A}_{1}$ \\
\hline 3 & $\mathrm{~A}_{3}$ \\
\hline
\end{tabular}

Fuente: Elaboración propia, 2020.

Los resultados obtenidos presentan la clasificación final del ordenamiento del destino turístico mejor clasificado; en este sentido, destaca la alternativa como el de mayor competitividad a la alternativa $A 2$, seguido por la $A 1$ y por último la A3. Adicionalmente, tomando en consideración el número de variables de cada criterio, se realizaron los cálculos de manera individual para analizar la competitividad a cada alternativa, considerando que para cada criterio existen diferentes pesos; los resultados se muestran en el cuadro 7.

Cuadro 7

Ranking individual de alternativas según criterios

\begin{tabular}{|c|c|c|c|c|}
\hline Posición & $\begin{array}{c}\text { Gestión del } \\
\text { Destino }\end{array}$ & $\begin{array}{c}\text { Factores y } \\
\text { Recursos de } \\
\text { Atracción }\end{array}$ & $\begin{array}{c}\text { Factores } \\
\text { Ambientales }\end{array}$ & $\begin{array}{c}\text { Factores } \\
\text { Sociales }\end{array}$ \\
\hline 1 & A2 & A1 & A2 & A2 \\
\hline 2 & A1 & A2 & A1 & A1 \\
\hline 3 & A3 & A3 & A3 & A3 \\
\hline
\end{tabular}

Fuente: Elaboración propia, 2020.

Como bien se observa, el destino A2 es el que se encuentra mejor posicionado en los criterios de Gestión del Destino, Factores Ambientales y Factores Sociales. Al respecto, Davenport y Prusak (1998) mencionan la relevancia de la valoración de este recurso intangible para este tipo de destinos, de ahí que el MCDA apoye a la toma de decisiones en un contexto real (León y Larrañaga, 2019). 
En última instancia, quien toma las decisiones es quien hará una evaluación de si la recomendación presentada está de acuerdo con sus preferencias o no, pero con esta metodología diversos problemas del mundo real pueden ser abordados y obtener una buena recomendación.

\section{Conclusiones}

El actual entorno globalizador y competitivo, sumado al desarrollo de la economía de los destinos turísticos, los llevan a buscar nuevos métodos que permitan conocer cuáles son las mejores estrategias implementadas que pudieran aprovecharse como parte de sus políticas para el desarrollo del turismo.

Por tanto, este trabajo presenta un análisis empírico del conocido modelo de Crouch-Ritchie (2010) para determinar la competitividad de tres destinos de sol y playa ubicados en el Caribe Colombiano, como son Santa Martha, Cartagena y San Andrés, para lo cual se consideraron las dimensiones política, económica, ambiental y social, incluyendo en ellas 87 indicadores con los que se evaluaron las tres ciudades. Este análisis se puede considerar como un procedimiento prototipo para utilizar el modelo de Crouch-Ritchie basado en los problemas multicriterio para la evaluación de la competitividad de los destinos turísticos en Colombia.

Cabe mencionar que, en el momento de la integración de los datos para las variables individuales consideradas en el estudio, se presentó la dificultad de contar con una entidad que agrupara de manera estandarizada esta información; se observó que no hay uniformidad para la generación de datos en los destinos turísticos consultados. Por lo anterior, el procesamiento de los datos debieron sufrir modificaciones (normalización) por los investigadores para hacer comparables (SITUR, CITUR, DANE y otras fuentes oficiales consultados).

El beneficio de la identificación de la alternativa de decisión del destino turístico más competitivo a través del método multiatributo contribuye en las gestiones que realizan las entidades públicas y privadas, en este caso los departamentos, así como de las cámaras que participan en el turismo, para orientar al sector y, en su caso, promover la utilización de herramientas que faciliten la toma de decisiones de manera más objetiva y así generar estrategias encaminadas a lograr ventajas competitivas en la industria que se observa en crecimiento en este destino (León y Larrañaga, 2019).

Respecto a la revisión del estado del arte, los estudios realizados utilizando métodos multicriterios con experiencias similares en el turismo, se tienen como autores a León y Leyva (2017), Leyva, Gastélum y Urías (2016), Crouch-Ritchie (2010), León y Larrañaga (2019), permitieron descubrir que las aplicaciones basadas en este método, en particular ELECTRE III, se encuentran en investigaciones derivadas de problemas de la vida real, mismos que se hallan entre subjetividad y objetividad.

En este sentido, los resultados obtenidos, en orden ascendente, permitiendo evaluar que destacó Cartagena, como el que más y mejor ha logrado este resultado en comparación con Santa Martha y San Andrés en términos de competitividad, al haber obtenido las mejores posiciones en los criterios de gestión del destino, factores ambientales y factores sociales; Santa Martha se ubicó por arriba en el criterio de factores y recursos de atracción.

Finalmente, para obras futuras, el uso de este procedimiento podría utilizarse en otros destinos turísticos más competitivos entre ellos y así identificar qué factor es más atractivo para impulsar el turismo, con la hechura de mejores planes y alcanzar mejores tomas de decisión.

\section{Referencias bibliográficas}

Alireza, A., Majid, M., y Rosnah, M. (2010). Simple Additive Weighting Approach to Personnel Selection Problem. International Journal of Innovation. Management and Technology, 1(5), 511-515. 
Álvarez Carrillo, P. A., León Santiesteban, M., Gastélum Chavira, D. A., Vega Osuna, L. A. (2013). An Empirical Analysis of Competitiveness on Cities of Sinaloa, Mexico with an Outranking Method. Eureka-2013. Fourth International Workshop Proceedings. Published by Atlantis Press, 156-163.

Ascanio, A. (2008). Competencia turística y estrategias: la coherencia entre modelos. PASOS Revista de Turismo y Patrimonio Cultural, vol. 6, núm. 1, 117-120.

Barragán Muñoz, J. M. (2003). Medio ambiente y desarrollo en áreas litorales: introducción a la planificación y gestión integradas. Cádiz: Publicaciones de la Universidad de Cádiz, 301. <http://www.worldcat.org/title/medio-ambiente-y-desarrollo-en-areas-litorales-introduccion-a-laplanificacion-y-gestion-integradas/oclc/52141078. Beach Awards and Management>.

Benseny, G. y otros (2014). Neuquén, Universidad Nacional de Mar del Plata, Experiencias educativas: acciones de concientización turístico-ambiental en destinos costeros, 19. <http://170.210.83.98:8080/jspui/bitstream/123456789/536/1/56\%20Benseny\%20Padilla\%20et\%20al.pdf $>$.

Benzaquen, J. Del Carpio, L.A., Zegarra, L.A. y Valdivia, C.A. (2010). Un Índice Regional de Competitividad para un país, Revista Cepal, núm. 102, 69-86.

Bartolini, F., Gallerani, V., Samoggia, y Viaggi, D. (2005). Methodology for Multicriteria Analysis of agriEnvironmental Schemes. University of Bologna: Technical Report, Sixth Framework Programme.

Botero Saltarén, C.M. y otros (2008). Determinación de un sistema de calificación y certificación de playas turísticas-Informe final contrato 012. Santa Marta, Universidad del Magdalena, Santa Marta: Instituto de Investigaciones Tropicales.

Botero Saltarén, C.M. (2009). Tecnológica de Información y Gestión Centro ed. 4.La Habana, Cuba, s.n.Ciencia en su PC, 27-41. <http://www.redalyc.org/articulo.oa?id=181317813003>. VI Congreso Latinoamericano de Investigación Turística.

CagilabaVinau, RennieHamishG. Literature review of Beach Awards and Rating Systems. Environment Waikato Technical Report: TR05/24. [En línea] 0106 2005. [Citado el: 1508 2014]. <http://www.waikatoregion.govt.nz/Services/Publications/Technical-Reports/Literature-review-of-beachawards-and-rating-systems/>.

Carvajal, A. David, A. y otros (2003). Conceptos y guía metodológica para el manejo integrado de zonas costeras en Colombia, Manual 1: preparación, caracterización y diagnóstico. Santa Marta DTCH: Invermar , 2003 , Vol. Serie de Documentos Generales de INVEMAR núm. 12, 94.

CEPAL, Comisión Económica para América Latina y el Caribe. Estudio Económico de América Latina y el Caribe. CEPAL. [En línea] 2018. [Citado el 6 agosto 2019.] <https://repositorio.cepal.org/bitstream/handle/11362/43964 /141/S1800837_es.pdf>.

Certificación de playa ambiental para hoteles; manejo integrado. HuertaFrank2019, Revista Vinculando.

Cervantes Rosas, O.D. (2008). Diseño de un índice (VIP) para evaluar playas recreativas. Ensenada. Tesis doctoral inédita. Universidad de Baja California, 2008, 143.

Crouch, G.I. (2007). Modelling Destination Competitiveness. A Survey and Analysis of the Impact of Competitiveness Attributes. Queensland, Sustainable Tourism Pty Ltd, 54. 
Crouch, G.I. (2010). Destination Competitiveness: an Analysis of Determinant Attributes. Journal of Travel Research 2011, originally published online 19 march, 50-27.

Crouch, G.I. and Ritchie, J.R.B. (1999). Tourism, Competitiveness, and Social Prosperity. Journal of Business Research, 44, 137-52.

Diéguez Castrillón, I. y otros (2, agosto 2011). Cultur, 101 - 124. CULTUR, año 05, núm. 02 - Ago.

Díez Santos, D. (2012). La competitividad turística en espacios interiores: una propuesta metodológica para la determinación de competidores potenciales. Cuadernos de Turismo, núm. 30, 109-125.

Duque Brito, A. (2005). Turismo y competitividad en Venezuela: una aproximación a la situación actual venezolana. Visión Gerencial. Año 4, núm. 2, vol. 4, 133-150.

Dwyer, L. Mellor, R. Livaic, Z. Edwards, D. and Kim, C. (2004). Attributes of Destination Competitiveness: a Factor Analysis. Tourism Analysis, vol. 9, 1-11.

Dwyer, L. and Kim, C. (2003). Destination Competitiveness: Determinants and Indicators. Current Issues in Tourism, 369-414.

EcuRed (2020). Cartagena de Indias. Consultado el 23 de mayo de 2020. <https://www.ecured.cu/Cartagena_de_Indias_(Colombia)>.

Gomezelj Omerzel, D. (2006). Competitiveness of Slovenia as a Tourist Destination. Managing Global Transitions, núm. 2, vol. 4, 167-189.

Ibañes Reyna, I.A. (2012). Medio ambiente y política turística en México. Identidad territorial y promoción turística: la organización de eventos como estrategia de creación, consolidación y difusión de la imagen de marca del territorio. México: Ine Semarnat.

Jiménez Morales, M., de San Eugenio Vela, J. (2009). Las playas en la gestión sostenible del litoral.

Zer Revista de Estudios de Comunicación, 22

<https://www.researchgate.net/publication/44324601_Identidad_Territorial_y_Promocion_Turistica_La_ Organizacion_de_Eventos_Como_Estrategia_de_Creacion_Consolidacion_y_Difusion_de_la_Imagen_de_ Marca_Del_Territorio>.

James Rodney J. 43, 06 2000. Ocean \& Coastal Management, 495-514.

<https://www.researchgate.net/publication/222515644_From_beaches_to_beach_environments_linking _the_ecology_human-use_and_management_of_beaches_in_Australia>.

Krugman, P. (1994). Competitiveness: a Dangerous Obsession. Foreign Affairs, vol. 73, núm. 2. Nueva York: Council on Foreign Relations.

Labarca, N. (2008). Competitividad en el sector metalmecánico del estado Zulia. Formación Gerencial, Año 7, núm. 1, 51-69.

León Santiesteban, M. Flores Gamboa, S. y Leyva López, J.C. (2019). Determinación de la competitividad de los destinos turísticos bajo un enfoque multicriterio en el noroeste de méxico. un análisis comparativo 20152017. Revista Latinoamericana de Turismología / RELAT. Juiz de Fora, (Brasil), vol. 5, 1-11, Jan./ Dez.

León Santiesteban, M. y Larrañaga Núñez, A.M. (2019). Integración de conocimiento en restaurantes mediante el análisis multicriterio para la toma de decisiones. Inquietud Empresarial, vol. XIX, núm. (2). 19(2), 25-38. 
León Santiesteban, M. y Leyva López, J.C. (2017). A Multicriteria Decision aid for Evaluating the Competitiveness of Tourist Destinations in the Northwest of Mexico. Turismo y Sociedad, XXI, 51-67. DOI: <https://doi.org/10.18601/01207555.n21.03>.

León Santiesteban, M. y Leyva López, J.C. (2016). Metodología multicriterio para el análisis de la competitividad de los destinos turísticos. El caso del noroeste de México. México: Ediciones del Lirio.

Leyva López, J.C., Gastélum Chavira, D., Urías Ruiz, M. (2013). La aplicación de un enfoque multicriterio para comparar sectores económicos: el caso del Estado de Sinaloa, México: EMO Sheffield UK, 1-24.

Leyva, J. and Aguilera, M. (2005). A Multiobjective Evolutionary Algorithm for Deriving Final Ranking from a Fuzzy Outranking Relation. In: C. Coello Coello, E. Zitzler, A. Hernández (eds.), Evolutionary Multi-Criterion Optimization. Berlin Heidelberg: Third International Conference, EMO 2005, Lecture Notes in Computer Science 3410 Springer,.

Leyva, J.C. and Fernández, E. (2003). A New Method for Group Decision Support Based on ELECTRE-III Methodology. European Journal of Operational Research, 148, 1.

Mariño Jiménez, J.P. (2019). La certificación de playas como factor de competitividad para destinos turísticos costeros. El caso de la playa Gaviotas en Mazatlán Sinaloa. s.l.:Editorial Académica Española, 344. ISBN-13:978-620-0-04044-2 ISBN-10:6200040443.

MINCIT, Ministerio de Comercio, Industria y Turismo Resultados para el turismo para el año 2018. MINCIT (2018). [En línea] 2018. [Citado el 8 agosto 2019. <http://www.mincit.gov.co/getattachment/estudioseconomicos/estadisticas-e-informes/informes-de-turismo/2018/oee-vt-turismo-2018-28-032019.pdf.aspx>.

Nelson Cy otros (1, 01 2000). Ocean \& Coastal Management, vol. 43, 87-98. <http://www.sciencedirect.com/science/article/pii/S096456919900068X>.

OMT, Organización Mundial del Turismo (2018). Panorama OMT del turismo internacional. UNWTO. [En línea] [Citado el 4 agosto 2019]. <https://www.e-unwto.org/doi/pdf/10.18111/9789284419890?download=tru> . Panorama омт del Turismo Internacional. Edición 2017. UNWTO. [En línea]. 4 agosto, 2017. <https://www.e-unwto.org/doi/pdf/10.18111/9789284419043>. Resultados del Turismo Internacional 2018 y Perspectivas 2019. UNWTo. [En línea] 2019. [Citado el 5 agosto 2019. <http://cf.cdn.unwto.org/sites/all/files/pdf/unwto_barometer_jan19_presentation_sp.pdf>.

PARES Invirtiendo en el futuro. Suplemento de construcción de paz para el sector de turismo en Colombia. Fundación Paz \& Reconciliación. [En línea] 2018. [Citado el 8 agosto, 2019]. $<$ http://www.mincit.gov.co/minturismo/eventos-y-publicaciones/publicaciones/guias-turisticas-decolombia/inviertiendo-en-el-futuro.aspx>. ISBN: 978-958-56540-0-6. The Competitive Destination.

RitchieJ, R.B., Crouch G. (I. 21, 2000). Tourism Management, 1-7. Utilidad de los esquemas de certificación de playas para el manejo integrado costero. Evaluación de ocho certificaciones en Iberoamérica.

Peng, K. y Tzeng, G. (2012). Intelligent Decision Technologies. In: J. Watada, T. Watanabe, G. Phillips-Wren, R. Howlett, L. Jain (eds.), Strategies for Promoting Tourism Competitiveness Using a Hybrid MCDM Model, 107115, Springer Berlin Heidelberg. 
Pérez Contreras, E.O., Leyva López, J.C., Castillo Ortiz, M.G. y Zazueta Hernández, M.M., Figueroa Pérez J.F. (2018). Análisis comparativo de la competitividad de los destinos turísticos Querétaro y Guanajuato bajo un enfoque multicriterio. Teoría y Praxis, núm. 26, septiembre-diciembre, 153-186.

Roy, B. (1990). The Outranking Approach and the Foundations of ELECTRE methods.

Porter, M.E. (1991). Towards a Dynamic Theory of Strategy. Strategic Management Journal, 12 (Winter Special Issue), 95-117.

Porter, M. (1998). La ventaja competitiva de las naciones. Buenos Aires: Vergara.

Ritchie, J.R.B. and Crouch, G.I. (2003). The Competitive Destination: a Sustainable Tourism Perspective. Wallingford, UK: CABI.

Roger, M., Bruen, M. Maystre, L. (2000). ELECTRE and Decision Support. Methods and Applications in Engineering and Infrastructure Investment. New York: Springer Us.

Situr Santa Marta-Magdalena. Indicadores. Consultado el 30 de marzo de 2020. $<$ https://www.siturmagdalena.com/indicadores/secundarios $>$.

San Andrés, Gobernación del Archipiélago. Territorio. Consultado el 26 de mayo de 2020. <https://sanandres.gov.co/index.php/archipielago/mapas/territorio>.

San Andrés, Gobernación del Archipiélago. Anuario Estadístico 2018. Consultado el 26 de mayo de 2020. <https://www.sanandres.gov.co/index.php/gestion/planeacion/plan-de-desarollo/estadisticas/10909anuario-estadistico-2018/file>.

Visita Colombia Co. Santa Marta. Consultado el 26 de mayo de 2020c. <https://colombia.travel/es/san-andresisla>.

Visita Colombia Co. Cartagena de Indias. Consultado el 26 de mayo de 2020a. <https://colombia.travel/es/cartagena-de-indias>.

Visita Colombia Co. Santa Marta. Consultado el 26 de mayo de 2020b. <https://colombia.travel/es/santamarta>.

WEF World Economic Forum The Travel \& Tourism Competitiveness Report 2015. WEF. [En línea] 2015. [Citado el 5 Mayo 2019.] <http://www3.weforum.org/docs/TT15/WEF_Global_Travel\&Tourism_Report_2015.pdf>.

WEF World Economic ForumThe Travel \& Tourism Competitiveness Report 2007. Tourism Generis. [En línea] 2007. [Citado el 2 mayo 2019.] <https://www.tourism-generis.com/_res/file/4247/49/0/WEF_Travel TourismCompetitiveness_Report_2007.pdf $>$.

WEF WorldEconomic ForumThe Travel \& Tourism Competitiveness Report 2019. WEF. [En línea] 2019. [Citado el 5 mayo 2019]. <http://www3.weforum.org/docs/WEF_TTCR_2019.pdf>

Yepes Piqueras,V. 4.Murcia, Universidad de Murcia, julio-diciembre 1999, Cuadernos de Turismo, 89- 10. <http://revistas.um.es/index.php/turismo/article/view/22881>.

Esta obra está bajo una Licencia Creative Commons Attribución-NoCommercial 4.0 International

(cc) EY-NO 